\title{
Detection of circulating tumor cells (CTCs) in cerebrospinal fluid of a patient with HER2-overexpressing gastric cancer and single cell analysis of intra-patient heterogeneity of CTCs
}

\author{
Jang Ho Cho", Sun Young Kim", Jeeyun Lee, Se Hoon Park, Joon Oh Park, Young Suk Park, \\ Ho Yeong Lim, Won Ki Kang, Seung Tae Kim
}

Division of Hematology-Oncology, Department of Medicine, Samsung Medical Center, Sungkyunkwan University School of Medicine, Seoul, Republic of Korea

Contributions: (I) Conception and design: J Lee, ST Kim; (II) Administrative support: J Lee, ST Kim; (III) Provision of study materials or patients: All authors; (IV) Collection and assembly of data: All authors; (V) Data analysis and interpretation: JH Cho, SY Kim, J Lee, ST Kim; (VI) Manuscript writing: All authors; (VII) Final approval of manuscript: All authors.

\#These authors contributed equally to this work.

Correspondence to: Seung Tae Kim, MD. Division of Hematology-Oncology, Department of Medicine, Samsung Medical Center, Sungkyunkwan University School of Medicine, 81 Irwon-ro, Gangnam-gu, Seoul 135-710, Republic of Korea. Email: seungtae1.kim@samsung.com.

Background: The purpose of this study was to identify circulating tumor cells (CTCs) through single cell sorting using DEPArray technology in cerebrospinal fluid (CSF) samples from an advanced gastric cancer (AGC) patient with leptomeningeal seeding. We also demonstrated intra-patient heterogeneity of CTCs isolated from CSF samples at the single cell level.

Methods: We used the DEPArray system to identify and align single enabled CSF and CTCs based on a multi-parallel fluorescence analysis. The CSF samples were stained with an antibody cocktail recognizing DAPI, cytokeratin, EpCAM, and HER2. The stained cells were sorted and identified into a single, variable cell based on a multiparametric fluorescence analysis. The CSF and CTC subpopulations were quantified for absolute cell counts and relative frequency. Based on the stained morphology of each single CSF and CTC, five groups were identified.

Results: We stratified the CTCs to evaluate the character of the CTCs isolated from the CSF, according to CK, EpCAM and HER2. In total, 78.2\% of the CTCs isolated from the CSF showed HER2 overexpression, which was consistent with the status of HER2 in the patient's primary gastric tumor. The most common types were HER2 positive, CK positive, and EpCAM positive CTCs (56.6\%). On the other hand, 21.8\% of the CTCs isolated from the CSF did not show HER2 overexpression, which was inconsistent with the primary tumor. Her2 negative, CK negative, and EpCAM positive CTCs were the predominant types among the CTCs without HER2 overexpression (19.7\%).

Conclusions: We enriched the CTCs through a single cell sorting process with DEPArray technology in CSF samples of an AGC patient with leptomeningeal seeding. We also demonstrated intra-patient heterogeneity of the CTCs at the single cell level.

Keywords: Advanced gastric cancer (AGC); circulating tumor cells (CTCs); cerebrospinal fluid (CSF)

Submitted Apr 16, 2019. Accepted for publication Aug 19, 2019.

doi: $10.21037 /$ tcr.2019.09.27

View this article at: http://dx.doi.org/10.21037/tcr.2019.09.27 


\section{Introduction}

Cancer is an evolutionary process in which differences in micro-environmental selection stressors and iatrogenic intervention create dynamic cellular changes that, over time, continually generate variant subpopulations that systemically spread (1). According to the clonal theory of tumor evolution, cancer is an evolving process (2), and certain stressors exerted by multiple lines of treatment may lead to many more aggressive subclone populations or even those with an acquired drug resistance (1). Liquid biopsy using circulating tumor cells (CTCs) is one of the most sensitive approaches to monitor tumor molecular evolution $(3,4)$. Molecular characterization of CTCs is important to evaluate intra-patient tumor heterogeneity and to determine the tumor evolution. CTCs are rare and very heterogeneous populations of tumorigenic cancer cells in the blood of patients with cancer, which complicates their isolation and characterization. DEPArray (Silicon Biosystems, Italy) is a dielectrophoresis-based method used in the isolation and molecular characterization of single tumor cells, including CTCs (5-8). This device analyzes and sorts single and rare cells using cell entrapment inside dielectrophoretic cages and image-based selection processes. Each selected cell can be moved by software-controlled electrical fields and eventually isolated downstream for molecular analysis (7).

Advanced gastric cancer (AGC) metastasizes to the central nervous system (CNS) in less than $5 \%$ of patients $(9,10)$. However, it has a devastating prognosis and lacks an efficient and standard treatment approach. In AGC patients with CNS metastasis, the role of human epidermal growth factor receptor 2 (HER2) is unknown. HER2 is an important biomarker and key driver of tumorigenesis in AGC, with studies reporting amplification or overexpression in $10-22 \%$ of tumors $(11,12)$. The ToGA trial demonstrated a significant survival benefit of trastuzumab in patients with HER2-overexpressing AGC (13). But, that study did not include patients with brain metastasis. The differences between HER2 positivity in primary and metastatic cancer can be partially explained by intratumor heterogeneity. Only a few studies have reported HER2 status for both primary gastric cancer and CNS metastasis, because it is difficult to obtain cancer tissue of CNS metastasis.

In this study, we identified CTCs through a single cell sorting process with DEPArray technology from cerebrospinal fluid (CSF) samples in a patient with leptomeningeal seeding from AGC. We also demonstrated intra-patient heterogeneity of CTCs isolated from CSF at the single cell level.

\section{Methods}

\section{A patient}

This investigation was conducted in accordance with the ethical standards of the Declaration of Helsinki and national and international guidelines and was approved by the Institutional Review Board at Samsung Medical Center. Between October 2013 and January 2016, patients with gastrointestinal cancer, rare cancer, and lung cancer were prospectively enrolled in the SMC Oncology Biomarker study. The sample-acquisition protocol was performed for eligible patients. Tumor samples and liquid samples from patients who provided written informed consent were prepared for the clinical and molecular studies.

\section{Sample collection from CSF}

Serial samples from CSF were collected at day $0,1,3,6$, and 10 from a HER2-positive AGC patient with leptomeningeal seeding. These serial CSF samplings were conducted to help avoid increased intracranial pressure. CSF samplings were conducted through the Ommaya. Total volume of samples was $280 \mathrm{~mL}$. After cytological confirmation by a pathologist, the samples were centrifuged for 5 minutes at $400 \times \mathrm{g}$ at room temperature and then were analyzed for CTC detection using the DEPArray system.

\section{CTC detection using the DEPArray from CSF samples}

The samples were washed with running buffer (Miltenyi Biotec) and added to Inside Perm solution (Miltenyi Biotec). After 10 minutes, the cells were stained with cytokeratin (CK) [PE conjugated pan cytokeratin antibody (C-11); Abcam], EpCAM (APC conjugated CD326 EpCAM human antibody; Miltenyi Biotec), and HER2 [rabbit polyclonal $\mathrm{Neu}(\mathrm{C}-18)$ antibody; Santa Cruz] and labeled with using Alexa Fluor 488 dye (Invitrogen).

After fixation with $2 \%$ paraformaldehyde, cell sorting experiments were performed by DEPArray (Silicon Biosystems) as described in the manufacturer's instructions. In short, DEPArray cartridges (A300K) were manually loaded with $14 \mu \mathrm{L}$ of sample (30,000 cells) and $830 \mu \mathrm{L}$ of SB buffer. After loading the cartridge into the DEPArray system, the sample was injected into a microchamber, where the cells were exposed to an electric field consisting of 16,000 electrical cages in which the individual cells could be trapped. Image frames for each of the four fluorescent filters (FITC, PE, APC, and DAPI) and bright-field 

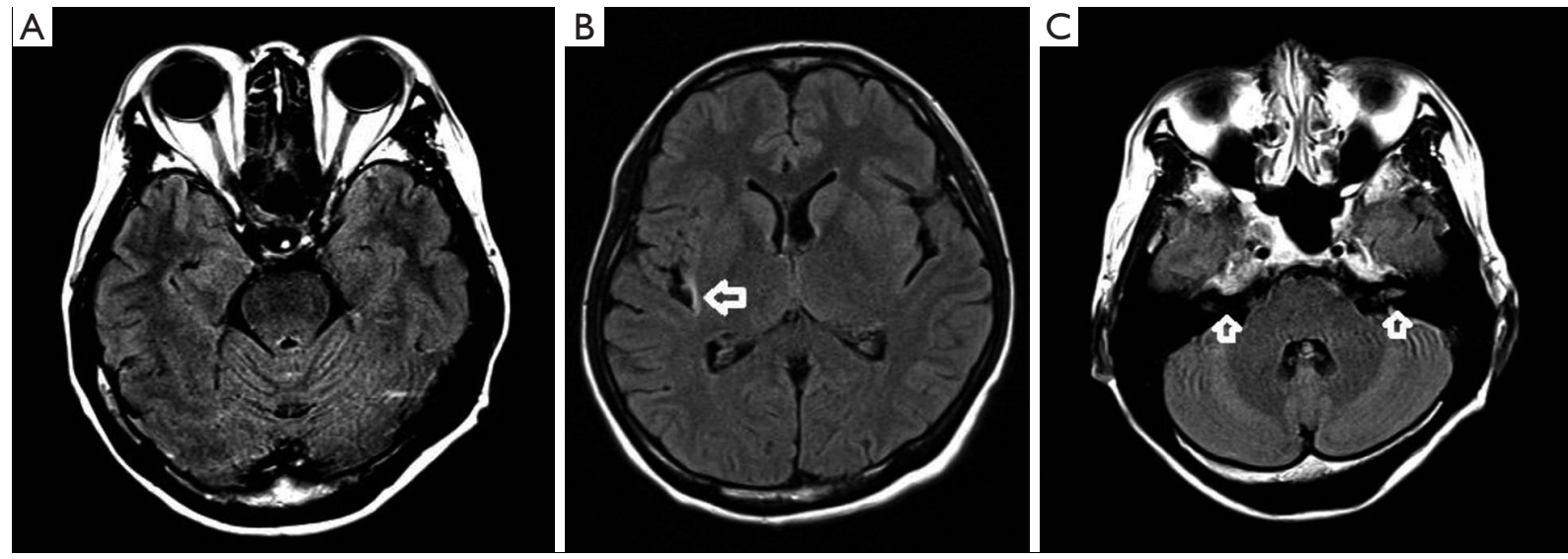

Figure 1 Brain magnetic resonance imaging of a patient with leptomeningeal seeding from gastric cancer. Axial contrast-enhanced FLAIR MR image shows along the (A) cerebellar folia, (B) right sylvian fissure, and (C) both internal auditory canal.

images were captured. Cell detection was based on a DAPI fluorescence threshold. For each cell, a unique ID was assigned. Captured images were processed and presented by the CellBrowser software that enabled the selection of cells of interest by the operator. Nucleated CK/EpCAM/HER2 stained cells were chosen independently based on single or double positive expression and were moved to a parking area in the cartridge. Individual cells were then moved to a recovery area where a last visual confirmation of cell presence was performed. It takes about 4 hours to isolate the single tumor cells (CK/EpCAM positive) via DEPArray loading, and more details are in the Figure S1.

\section{Results}

\section{Patient}

A 43-year-old woman initially presented in 2013 with stage IV GC with peritoneal seeding and distant lymph node metastasis. Endoscopic biopsy was conducted on a primary gastric cancer lesion, and the biopsied tumor sample showed HER2 3+ by immunohistochemistry (IHC). As first-line therapy, the patient received chemotherapy including HER2-targeted agent (trastuzumab in combination with capecitabine and cisplatin), and the progression-free survival (PFS) for first-line therapy was 11 months. Subsequently, irinotecan as second-line therapy and docetaxel as third-line therapy were administered. In November 2016, the patient suffered from headache and vomiting, and her brain was examined by magnetic resonance imaging (MRI). The MRI revealed leptomeningeal seeding (Figure 1), and the patient received an Ommaya reservoir for diagnosis and treatment. Cytology of CSF confirmed metastatic adenocarcinoma. Five serial CSF samplings were conducted through the Ommaya reservoir, which help to prevent increased intracranial pressure.

\section{Identification of CTC in CSF}

A schematic workflow for isolation of CSF CTC is shown in Figure S1. Samples of CSF were stained for 4 markers (DAPI, CK, EpCAM, and HER2). These stained cells were sorted and identified based on a multiparametric fluorescence analysis through the DEPArray system (Cellbrowser-software). Total cells $(/ \mathrm{mL})$ were counted in five serial CSF samples collected at days 0, 1, 3, 6, and 9 (Figure 2A). Among the total cells, CTCs were resorted and identified based on staining of at least one marker among CK, EpCAM, and HER2. Figure $2 B$ reveals individual CTCs stained with CK, EpCAM, and HER2 isolated through the DEPArray system.

\section{Heterogeneity of CTCs isolated from CSF}

To evaluate the character of the CTCs isolated from CSF, we stratified the CTCs according to CK, EpCAM, and HER2 expression (Figure $3 A, B$ ). In total, $78.2 \%$ of the CTCs isolated in the CSF showed HER2 overexpression, consistent with the status of HER2 in the patient's primary tumor. HER2 positive, CK positive, and EpCAM positive CTC were the most common type of CTCs (56.6\%). Meanwhile, $21.8 \%$ of the CTCs isolated in the CSF showed no HER2 overexpression. Among the CTCs without HER2 overexpression, HER2 negative, CK negative, and EpCAM 
A

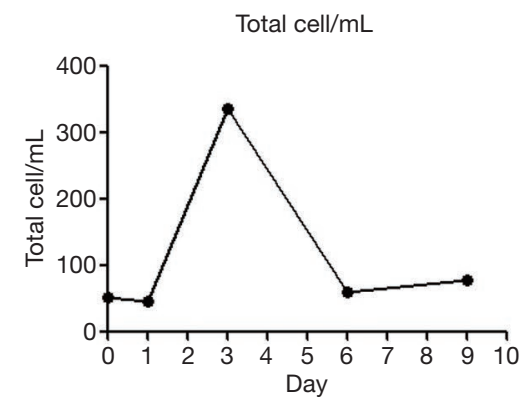

$\mathrm{CK} / \mathrm{EpCam}+\mathrm{cell} / \mathrm{mL}$

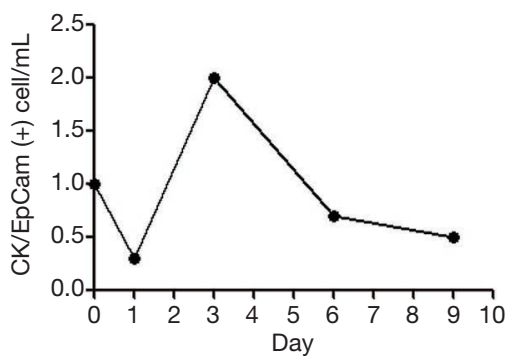

\begin{tabular}{ccc}
\hline Date & Total cell/mL & CK/EpCam positive cell/mL \\
\hline 20161128 & 52.9 & 1.0 \\
20161129 & 46.2 & 0.3 \\
20161202 & 335.8 & 2.0 \\
20161205 & 60.0 & 0.7 \\
20161208 & 78.2 & 0.5 \\
\hline
\end{tabular}

B

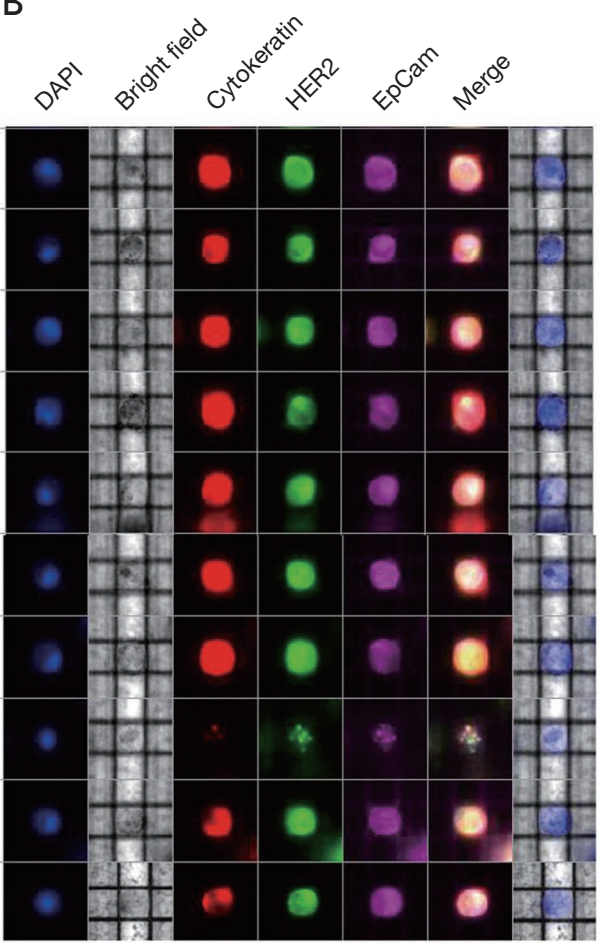

Figure 2 Detection of cerebrospinal fluid (CSF) circulating tumor cells (CTCs) in a gastric cancer patient. (A) Individual numbers of total cells and tumor cells in CSF detected using the DEPArray from the patient during follow-up. (B) Gallery of individual isolated CSF CTCs from a patient with leptomeningeal seeding from gastric cancer.

A

\begin{tabular}{|c|c|c|c|c|}
\hline HER2 & Cytokeratin & EpCam & Cell \# & $\% /$ total cell \\
\hline \multirow{4}{*}{ Positive } & Positive & Positive & 135 & 56.7 \\
\hline & Positive & Negative & 2 & 0.8 \\
\hline & Negative & Positive & 49 & 20.6 \\
\hline & Negative & Negative & 0 & 0.0 \\
\hline \multirow{3}{*}{ Negative } & Positive & Positive & 5 & 2.1 \\
\hline & Positive & Negative & 0 & 0.0 \\
\hline & Negative & Positive & 47 & 19.7 \\
\hline
\end{tabular}

B

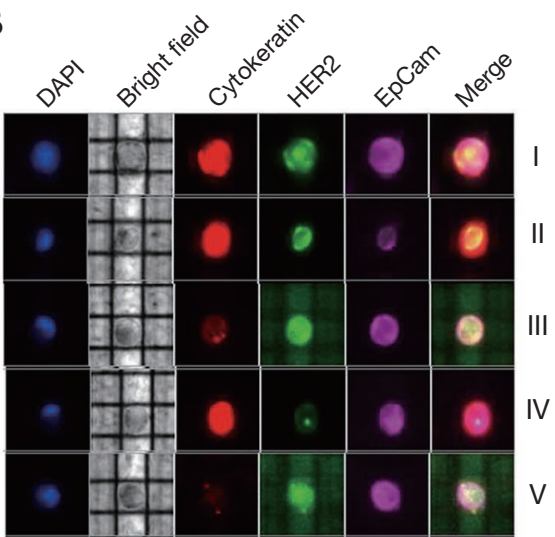

Figure 3 Characterization of HER2 positive CSF CTCs in a gastric cancer patient. (A) Quantification of subpopulations that expressed distinguished markers. I: CK+/EpCAM+/HER2+, II: CK+/EpCAM-/HER2+, III: CK-/EpCAM+/HER2+, IV: CK+/EpCAM+/HER2-, V: CK-/EpCAM+/HER2-. (B) Collection of validated single cell images (cytokeratin +/-, EpCAM +/-, and HER $2+/-$ ) of 5 groups in the CSF after absolute cell counting. CSF, cerebrospinal fluid; CTC, circulating tumor cell. 
positive CTCs were the predominant types (19.7\%).

\section{Discussion}

CTCs are rare and highly heterogeneous types of tumorigenic cancer cells in the blood of patients with metastatic cancer and might be an important consideration for precision medicine and personalized treatments $(14,15)$. In this study, we enriched CTCs through a single cell sorting process with DEPArray technology in CSF samples of a patient with leptomeningeal seeding from AGC. We also demonstrated the intra-patient heterogeneity of CTCs isolated from CSF. Moreover, although $78.2 \%$ of CTCs isolated in CSF showed HER2 overexpression, which was consistent with the status of HER2 in the patient's primary tumor, $21.8 \%$ of the isolated CTCs revealed a HER2 status inconsistent with the primary tumor.

The DEPArray technology is an ideal system to collect pure CTCs for further molecular characterization (16-18). Many studies have focused on metastatic breast cancer and single cell levels in CTCs using the DEPArray. Pestrin et al. reported heterogeneity of the PIK3CA mutational status within single CTCs in metastatic breast cancer patients (19). Bulfoni et al. used the DEPArray to identify and sort single and variable epithelial cells, such as CTCs and CTCs in the epithelial to mesenchymal transition (20). Gastric cancer is a very heterogeneous disease. The intratumor heterogeneity of GC is the major obstacle that hinders a positive outcome of anti-cancer treatment. Thus, the DEPArray might be useful to resolve the problem of heterogeneity in AGC. To the best of our knowledge, this study was the first to analyze the heterogeneity in single CTCs isolated from a patient with AGC.

HER2, a proto-oncogene, is an important prognostic biomarker and the key driver of tumorigenesis in GC. HER2 gene amplification and/or protein overexpression are found in $7-34 \%$ primary tumors (21-23). HER2 status assessment is essential in metastatic GC patients to select the specific subgroups that can benefit from a HER2 directed agent. Generally, HER2 is only evaluated in the primary lesion. Several studies have reported discordance of HER2 status in primary lesions and their corresponding metastatic lesions (24-27). However, the relationship between the primary lesion and the metastatic lesion has not been fully clarified. We evaluated the HER2 status of a metastatic lesion, at the single CTC level, compared to that of the primary lesion. Most CTCs (78.2\%) isolated from a metastatic lesion had concordance of HER2 status in the primary tumor. However, $21.8 \%$ of CTCs showed discordance of HER2 status in the primary tumor. This heterogeneity of HER2 status at the single CTC level might explain the limited efficacy of HER2 directed therapy.

Our findings are limited because they are based on a single case. Thus, a study with a large patient population for heterogeneity using the DEPArray in AGC is necessary. Nevertheless, this study revealed that the DEPArray analysis may be useful method to identify CTCs through single cell sorting and to evaluate the intra-patient heterogeneity of CTCs at the single cell level. Furthermore, the application of the DEPArray in tumor samples or liquid biopsy samples may be useful to evaluate the heterogeneity of a tumor.

\section{Acknowledgments}

Funding: This work was supported by a grant from the Korean Health Technology R\&D Project and the Ministry of Health \& Welfare, Republic of Korea (HI14C3418 HI14C2750), and by the Ministry of Education (NRF2018R1D1A1B07048349). Support was also provided by a grant (GF01140111) from Samsung Medical Center.

\section{Footnote}

Conflicts of Interest: All authors have completed the ICMJE uniform disclosure form (available at http://dx.doi. org/10.21037/tcr.2019.09.27). The authors have no conflicts of interest to declare.

Ethical Statement: The authors are accountable for all aspects of the work in ensuring that questions related to the accuracy or integrity of any part of the work are appropriately investigated and resolved. This investigation was conducted in accordance with the ethical standards of the Declaration of Helsinki and national and international guidelines and was approved by the Institutional Review Board at Samsung Medical Center. Written informed consent was obtained from all patients.

Open Access Statement: This is an Open Access article distributed in accordance with the Creative Commons Attribution-NonCommercial-NoDerivs 4.0 International License (CC BY-NC-ND 4.0), which permits the noncommercial replication and distribution of the article with the strict proviso that no changes or edits are made and the original work is properly cited (including links to both the formal publication through the relevant DOI and the license). See: https://creativecommons.org/licenses/by-nc-nd/4.0/. 


\section{References}

1. Greaves M, Maley CC. Clonal evolution in cancer. Nature 2012;481:306-13.

2. Marjanovic ND, Weinberg RA, Chaffer CL. Cell plasticity and heterogeneity in cancer. Clin Chem 2013;59:168-79.

3. Marchetti A, Del Grammastro M, Felicioni L, et al. Assessment of EGFR mutations in circulating tumor cell preparations from NSCLC patients by next generation sequencing: toward a real-time liquid biopsy for treatment. PLoS One 2014;9:e103883.

4. Shaw JA, Page K, Blighe K, et al. Genomic analysis of circulating cell-free DNA infers breast cancer dormancy. Genome Res 2012;22:220-31.

5. Shim S, Stemke-Hale K, Tsimberidou AM, et al. Antibodyindependent isolation of circulating tumor cells by continuousflow dielectrophoresis. Biomicrofluidics 2013;7:11807.

6. Gupta V, Jafferji I, Garza M, et al. ApoStream( $\left.{ }^{\mathrm{TM}}\right)$, a new dielectrophoretic device for antibody independent isolation and recovery of viable cancer cells from blood. Biomicrofluidics 2012;6:24133.

7. Fabbri F, Carloni S, Zoli W, et al. Detection and recovery of circulating colon cancer cells using a dielectrophoresisbased device: KRAS mutation status in pure CTCs. Cancer Lett 2013;335:225-31.

8. Peeters DJ, De Laere B, Van den Eynden GG, et al. Semiautomated isolation and molecular characterisation of single or highly purified tumour cells from CellSearch enriched blood samples using dielectrophoretic cell sorting. Br J Cancer 2013;108:1358-67.

9. Wadhwa R, Taketa T, Correa AM, et al. Incidence of brain metastases after trimodality therapy in patients with esophageal or gastroesophageal cancer: implications for screening and surveillance. Oncology 2013;85:204-7.

10. Blay C, Chiforeanu DC, Boucher E, et al. Incidence of brain metastases in HER2 + gastric or gastroesophageal junction adenocarcinoma. Acta Oncol 2015;54:1833-5.

11. Van Cutsem E, Bang YJ, Feng-Yi F, et al. HER2 screening data from ToGA: targeting HER2 in gastric and gastroesophageal junction cancer. Gastric Cancer 2015;18:476-84.

12. Warneke VS, Behrens HM, Böger C, et al. Her2/neu testing in gastric cancer: evaluating the risk of sampling errors. Ann Oncol 2013;24:725-33.

13. Bang YJ, Van Cutsem E, Feyereislova A, et al. Trastuzumab in combination with chemotherapy versus chemotherapy alone for treatment of HER2-positive advanced gastric or gastro-oesophageal junction cancer (ToGA): a phase 3, open-label, randomised controlled trial. Lancet 2010;376:687-97.

14. Schork NJ. Personalized medicine: Time for one-person trials. Nature 2015;520:609-11.

15. Pantel K, Alix-Panabières C. Real-time liquid biopsy in cancer patients: fact or fiction? Cancer Res 2013;73:6384-8

16. Vishnoi M, Peddibhotla S, Yin W, et al. The isolation and characterization of CTC subsets related to breast cancer dormancy. Sci Rep 2015;5:17533.

17. Polzer B, Medoro G, Pasch S, et al. Molecular profiling of single circulating tumor cells with diagnostic intention. EMBO Mol Med 2014;6:1371-86.

18. De Luca F, Rotunno G, Salvianti F, et al. Mutational analysis of single circulating tumor cells by next generation sequencing in metastatic breast cancer. Oncotarget 2016;7:26107-19.

19. Pestrin M, Salvianti F, Galardi F, et al. Heterogeneity of PIK3CA mutational status at the single cell level in circulating tumor cells from metastatic breast cancer patients. Mol Oncol 2015;9:749-57.

20. Bulfoni M, Gerratana L, Del Ben F, et al. In patients with metastatic breast cancer the identification of circulating tumor cells in epithelial-to-mesenchymal transition is associated with a poor prognosis. Breast Cancer Res 2016;18:30.

21. Tanner M, Hollmén M, Junttila TT, et al. Amplification of HER-2 in gastric carcinoma: association with Topoisomerase IIalpha gene amplification, intestinal type, poor prognosis and sensitivity to trastuzumab. Ann Oncol 2005; 16:273-8.

22. Gravalos C, Jimeno A. HER2 in gastric cancer: a new prognostic factor and a novel therapeutic target. Ann Oncol 2008;19:1523-9.

23. Park DI, Yun JW, Park JH, et al. HER-2/neu amplification is an independent prognostic factor in gastric cancer. Dig Dis Sci 2006;51:1371-9.

24. Bozzetti C, Negri FV, Lagrasta CA, et al. Comparison of HER2 status in primary and paired metastatic sites of gastric carcinoma. Br J Cancer 2011;104:1372-6.

25. Kochi M, Fujii M, Masuda S, et al. Differing deregulation of HER2 in primary gastric cancer and synchronous related metastatic lymph nodes. Diagn Pathol 2013;8:191.

26. Shibata R, Nimura S, Hashimoto T, et al. Expression of human epidermal growth factor receptor 2 in primary and paired parenchymal recurrent and/or metastatic sites of gastric cancer. Mol Clin Oncol 2014;2:751-5.

27. Kim MA, Lee HJ, Yang HK, et al. Heterogeneous amplification of ERBB2 in primary lesions is responsible for the discordant ERBB2 status of primary and metastatic lesions in gastric carcinoma. Histopathology 2011;59:822-31.

Cite this article as: Cho JH, Kim SY, Lee J, Park SH, ParkJO, Park YS, Lim HY, Kang WK, Kim ST. Detection of circulating tumor cells (CTCs) in cerebrospinal fluid of a patient with HER2-overexpressing gastric cancer and single cell analysis of intra-patient heterogeneity of CTCs. Transl Cancer Res 2019;8(5):2107-2112. doi: 10.21037/tcr.2019.09.27 


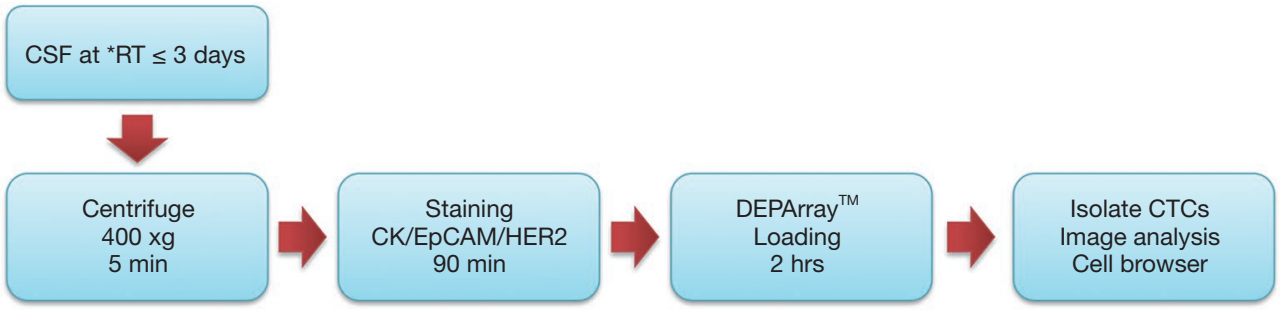

Figure S1 Schematic showing the workflow developed for the isolation of circulating tumor cells (CTCs) from cerebrospinal fluid (CSF). ${ }^{*} \mathrm{RT}$, room temperature. 